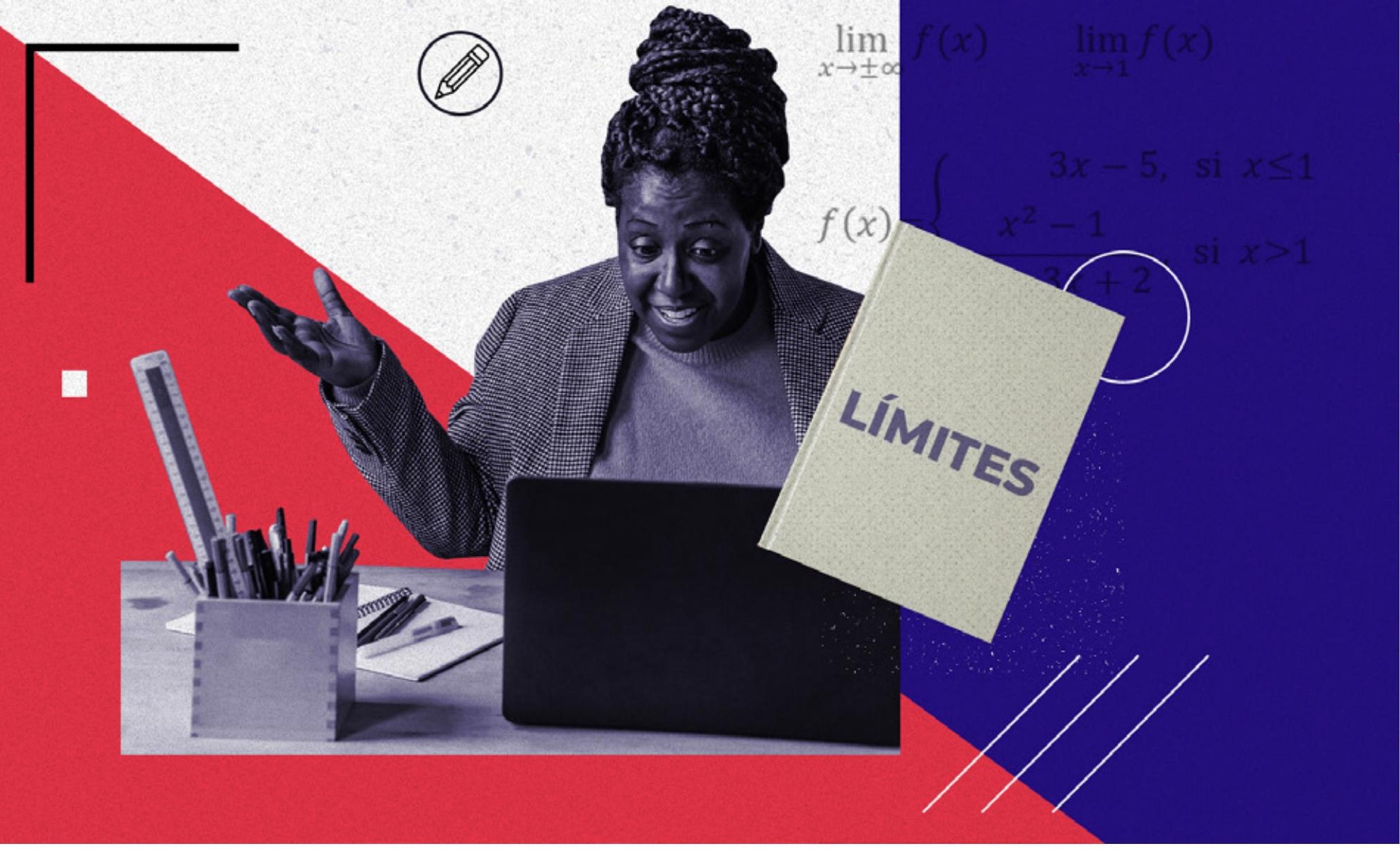

\title{
El uso de la tecnología en la enseñanza del límite para el fortalecimiento de competencias matemáticas en estudiantes de secundaria en tiempos de pandemia
}

THE USE OF TECHNOLOGY IN TEACHING THE LIMIT FOR THE STRENGTHENING OF MATHEMATICAL COMPETENCIES IN HIGH SCHOOL STUDENTS IN TIMES OF PANDEMIC

O USO DA TECNOLOGIA NO ENSINO DO LIMITE PARA O FORTALECIMENTO DAS COMPETÊNCIAS MATEMÁTICAS EM ALUNOS DO ENSINO MÉDIO EM TEMPOS DE PANDEMIA

Robinson Junior Conde-Carmona ${ }^{1}$; Andrés Antonio Fontalvo Meléndez ${ }^{2}$; Iván Andrés Padilla-Escorcia ${ }^{3}$ 


\section{Citar artículo como:}

Conde-Carmona, R.J., Fontalvo-Meléndez, A.A., y Padilla-Escorcia, I.A. (2021). El uso de la tecnología en la enseñanza del límite para el fortalecimiento de competencias matemáticas en estudiantes de secundaria en tiempos de pandemia. Educación y Ciudad, n. 41, pp. 147-170. https://doi.org/10.36737/01230425. $\mathrm{n} 41.2021 .2496$

Fecha de recepción: 22 de febrero de 2021

Fecha de aprobación: 6 de julio de 2021

\section{Resumen}

Este trabajo de investigación se desarrolló en tiempos de pandemia durante el año 2020, con el objetivo de fortalecer los procesos de aprendizaje del límite de una función y sus aplicaciones mediante una propuesta de intervención en la modalidad e-learning con el uso de herramientas TIC que permiten la GeoGebra y la mediación y el uso de plataformas virtuales como: Zoom, Google Meet y Google Classroom, entre otras, como medios de comunicación entre el investigador y los participantes. En esta propuesta se abordaron situaciones problemáticas que relacionaban el concepto de límite de manera dinámica, lo que permitió construir un desarrollo significativo de competencias matemáticas como la interpretación, la representación, la modelación y la resolución de problemas en los estudiantes de grado once de una institución educativa privada de la ciudad de Barranquilla.

Palabras clave: Competencias matemáticas; TIC; didáctica; formación de profesores.

\section{Abstract}

This research work was developed in times of the pandemic during 2020 , with the aim of strengthening the learning processes of the limit of a function and its applications through an intervention proposal in the e-learning modality with the use of ICT tools GeoGebra which lets the mediation and use of virtual platforms such as: Google Meet, Zoom, Google Classroom, among others as a means of communication between the researcher and the participants. In this proposal, problematic situations that related the concept of limit in a dynamic way were addressed, which made possible to build a significant development of mathematical competencies such as interpretation, representation, modeling, and problem solving in eleventh grade students from a private educational institution in the city of Barranquilla.

Keywords: Mathematical competences; ICT; Didactics; Teacher training.

\section{Resumo}

Este trabalho de investigação foi desenvolvido em tempos de pandemia durante 2020, com o objetivo de fortalecer os processos de aprendizagem do limite de uma função e suas aplicações através de uma proposta de intervenção na modalidade e-learning com a utilização de ferramentas TIC que permitem ao GeoGebra, mediação e utilização de plataformas virtuais como: Google Meet, Zoom, Google Classroom, entre outros, como meio de comunicação entre o pesquisador e os participantes. Nesta proposta foram abordadas situações problemáticas que relacionavam $o$ conceito de limite de forma dinâmica, o que permitiu a construção de um desenvolvimento significativo de competências matemáticas como interpretação, representação, modelação e resolução de problemas em alunos do $11^{\circ}$ ano de uma instituição. instituição na cidade de Barranquilla.

Palavras-chave: Competências matemáticas; TIC; didática; formação de professores. 


\section{Introducción}

$\mathrm{E}$ 1 docente de matemáticas es un facilitador que promueve prácticas capaces de fortalecer el desarrollo de competencias matemáticas mediante la creación de estrategias que contribuyen al cumplimiento de este objetivo en su quehacer. Un ejemplo de esto es el uso de la tecnología, un elemento fundamental en la constitución de estas estrategias. Esto dado su relevancia social en el campo educativo, siempre que los profesores hagan uso de estas de manera asertiva y con intencionalidades de tipo didáctico-pedagógica para la enseñanza de esta área del conocimiento (Valbuena y Conde-Carmona, 2018; Padilla-Escorcia y Conde-Carmona, 2020).

No obstante, la contingencia ocasionada por el COVID-19 ha generado daños a nivel mundial, de tipo económico, empresarial, laboral y educativo, entre otros. En el contexto educativo, la enseñanza en todos los niveles de escolaridad, tuvo que asumir una nueva modalidad de enseñanza de un momento a otro. Así, el e-learning tomó auge en las miles de escuelas y universidades que conforman la nación, así mismo, el uso de plataformas digitales por parte de las escuelas, como: Zoom, Teams, Google Meet, Skype, ya existentes, pero poco utilizadas en la educación presencial (Aznar-Sala, 2020).

$\operatorname{PhD}(\mathrm{c})$. en Educación Matemática; Especialista en Métodos Numéricos Aplicados; Licenciado en Matemática; Docente e Investigador de la Universidad del Atlántico. Correo electrónico: rjconde@mail.uniatlantico.edu.co

Maestría en Didáctica de las matemáticas; Especialista en Didáctica de las matemáticas; Licenciado en Matemáticas; Docente del American School. Correo electrónico: afontalvo1230@gmail.com
Esta situación condujo a que diversos países de América Latina replantearan los procesos académicos en las instituciones educativas y desarrollaran planes estratégicos para la educación con modalidad a distancia. Chile, diseñó el Plan de Acción por Coronavirus; Venezuela, el Plan Pedagógico de Orientación y Prevención; Costa Rica el Programa de Apoyo del Proceso Educativo a Distancia; Panamá estableció la estrategia la Estrella de la Educación no se detiene; Paraguay conformó el programa Plan de Educación en tiempos de Pandemia. En el caso de Colombia, se propuso la formación virtual gratuita apoyada por entidades universitarias de ámbito privado y soportado en la plataforma web "Aprender Digital” (UNESCO, 2020). Lo anterior, en búsqueda de transformar de manera interactiva las formas de aprendizaje de los estudiantes, así como las prácticas pedagógicas y estrategias didácticas utilizadas por los profesores colombianos (Departamento Nacional de Planeación, 2020).

Todo esto, debido a que la práctica pedagógica del profesor mediante el uso de las TIC permite orientar la construcción de conocimiento, de manera que el estudiante logre vivenciar una experiencia educativa que impacte y trascienda los límites del espacio virtual y que emerja en el contexto mundial actual. Esto trajo consigo el cambio de la modalidad netamente presencial a la virtualización, y a su

Magíster en Educación; Especialista en Estadística Aplicada., Licenciado en Matemáticas; Docente e Investigador de la Universidad del Atlántico. ORCID: https://orcid. org/0000-0003-1210-3712 Correo electrónico: iapadiIla@mail.uniatlantico.edu.co 
vez, a la deconstrucción tanto de actitudes, como de los usos y aprendizajes en torno a las TIC (Olivares-Parada, Olivares-Parada y Parada-Rico, 2021).

Ahora bien, en los procesos de enseñanza de las matemáticas -un área compleja de desarrollar en las escuelas, incluso en la presencialidad, debido a su nivel de abstracción- el grado de dificultad a causa de las circunstancias actuales ha aumentado (Conde-Carmona y Padilla-Escorcia, 2021). Algo interesante, pues durante la presencialidad, en muchas ocasiones, se utilizó la tecnología para mediar los contenidos de esta área en las escuelas, ya que se contaba con las herramientas o instrumentos que permitían la mediación de contenidos con la tecnología, y en dónde tanto profesores como estudiantes exploraban conceptos matemáticos, compartían ideas y aprendían juntos a través de la interacción recíproca con el medio tecnológico. Sin embargo, la imprevista implementación de la modalidad virtual en los distintos países del mundo ocasionó que la única conexión de los profesores con sus estudiantes se llevara a cabo por medio del teléfono (WhatsApp, llamadas telefónicas) y mediante plataformas digitales, con la diferencia que el mediador del proceso (profesor), se encontraba lejos desde casa (Cassibba et al., 2021; Aldon et al., 2021).

Por consiguiente, esto ha generado dificultad en el proceso de enseñanza-aprendizaje de las matemáticas en tiempos del COVID-19. En la educación media secundaria ha sido un reto para el profesorado que enseña esta área del conocimiento mediante plataformas digitales. Específicamente mediante unidades de observación no participantes, se pudo constatar que los estudiantes de undécimo grado del Colegio San José Hermanitas de la Asunción en Barranquilla, Colombia, presentaron dificultades para la comprensión de la definición formal de lí- mites. Estos manifestaron no comprender el concepto, el cuál era nuevo para ellos. En general, coincidieron que las variables que intervienen en esta definición como épsilon y delta, no tienen relación con el contexto, lo que lleva a la incomprensión de este contenido. Aquí se establece una relación con lo propuesto por Mattos (2018), en el sentido de que el concepto de límite es considerado una de las temáticas más complejas en la matemática, puesto que la abstracción que contiene en su desarrollo es muy alta; y es por esa razón que diversos investigadores se han enfocado en estudiar sobre el proceso cognitivo que desarrollan los estudiantes para comprender la definición formal de límite, difícilmente comprensible para los estudiantes, incluso en la modalidad presencial.

Por otra parte, Guerrero y Hernández (2020) mencionan que una de las dificultades en la comprensión del límite de una función, radica en que muchos docentes que hoy día enseñan este contenido, presentaron debilidades en los procesos de aprendizaje de este y de sus respectivas relaciones fenomenológicas cuando fueron maestros en formación inicial, y que esto, posteriormente repercutió en sus prácticas pedagógicas. Así pues, es necesario que el profesor desarrolle nuevos métodos de enseñanza encaminados a la creación e innovación de contenidos como la definición formal de límite de manera dinámica y más al alcance de los estudiantes. Ante esto, es de vital importancia el uso de estrategias y recursos que contribuyan de manera efectiva en la mediación de las TIC con el contenido matemático a plasmar mediante estos recursos (Jiménez y Galán, 2017).

Sin embargo, se debe tener en cuenta que bajo la educación e-learning es aún más complejo la constitución de estas estrategias en la enseñanza de conteni- 
dos complejos como el límite de una función. Pese a esto, se deben buscar alternativas que contribuyan a llevar a cabo la enseñanza bajo esta modalidad (Calvo, 2019; Failache, Katzkowicz y Machado, 2020). De este modo, toma relevancia el uso de herramientas tecnológicas con fines de enseñanza para el abordaje de conceptos abstractos en matemáticas, especialmente GeoGebra, software especializado del área, dada su dinamización y facilidad para representar los contenidos de una forma más visual, al alcance de los estudiantes y fundamental para la enseñanza de conceptos abstractos (Granados-Ortiz y Padilla-Escorcia, 2021).

En ese orden de ideas, y en búsqueda de fortalecer el aprendizaje de los estudiantes acerca del límite de una función, se plantea la siguiente pregunta-problema de investigación: ¿ cómo una propuesta didáctica y tecnológica facilita los procesos de aprendizaje del concepto de límite de una función por parte de estudiantes de undécimo grado en tiempos del COVID-19? Frente a ello, se pretende dar respuesta teniendo como objetivo general de la investigación: crear una propuesta didáctica y tecnológica que facilite el aprendizaje del concepto de límite de una función por parte de estudiantes de undécimo grado del Colegio San José Hermanitas de la Asunción en Barranquilla, Colombia, en tiempos del COVID-19.

\section{Antecedentes}

En primera medida, se tiene el trabajo realizado por Volverás-Espinosa (2015) titulado: "Propuesta didáctica para la enseñanza de límites de funciones en el grado undécimo de la I.E El Rosario integrando GeoGebra", cuyo objetivo consistía en que los estudiantes adquirieran una mejor comprensión del concepto de límites de funciones. Para esto, se diseñaron cuatro subtemas basados en clases teóricas y prácticas; estos son: Noción intuitiva de límite de funciones en un punto mediante la definición formal; visualización del concepto de límite de funciones en un punto; existencia del límite y visualización de límites laterales e indeterminaciones de la forma 0/0; y visualización de límites de funciones que involucran el infinito. Se concluyó que el abordaje de estos subtemas del límite de una función por medio de herramientas tecnológicas, facilitó la comprensión de estos conceptos. Además, el uso de computadores y del software educativo GeoGebra, generó interés y motivación en los estudiantes para el aprendizaje de estos contenidos, situación que mediante la enseñanza tradicional de estos contenidos (tablero y marcador) no había provocado este impacto en la comunidad educativa. Igualmente, se observó que un alto porcentaje de los estudiantes interpretó la definición de límite de funciones de forma gráfica mediante GeoGebra.

Así mismo, Trujillo, Vera y Saraza (2019) desarrollaron la investigación titulada: "Ingeniería didáctica como recurso metodológico para el aprendizaje de los conceptos de límite y continuidad", cuyo objetivo estuvo dirigido a la comprensión por parte de estudiantes sobre conceptos abstractos como el límite y la continuidad en maestros en formación inicial de la Licenciatura en Matemáticas de la Universidad Francisco de Paula Santander, Colombia, por medio de un diseño de secuencias didácticas, en las que se tomó como referencia los cuatro tipos de representación propuestos por Duval (1995): numérico, algebraico, gráfico y comunicativo.

Usualmente, la enseñanza del concepto de límite es abordada a través de la representación algebraica, omitiéndose representaciones que contribuyen en una comprensión más robusta de este concepto, 
como lo es el registro gráfico, y que conduce a que los estudiantes tengan dificultades para la comprensión y aplicación de este contenido. Con base en lo anterior, en este trabajo se destaca la importancia que tuvo la aplicación de secuencias didácticas para fortalecer y desarrollar los conocimientos de los estudiantes alrededor del concepto de límite mediante registros semióticos (lenguaje natural, tabular, gráfico y algebraico) (Duval, 1995; D’ Amore, 2009). De esta forma, el uso de diferentes representaciones para la resolución de problemas relacionados con límite de funciones permitió un mejor desempeño en los estudiantes; específicamente el $65 \%$ de ellos alcanzó un nivel alto cuando utilizó estos registros de representación, mientras que el 80\% alcanzó un nivel entre medio y bajo cuando solamente utilizó un registro en los ejercicios de límite de funciones. Es claro entonces que para enseñar el concepto del límite de una función, se requiere de innovación y creatividad en la formación de maestros en nivel inicial, dado su nivel de complejidad y abstracción. A su vez, los registros de representación plasmados de manera didáctica mediante software matemáticos como GeoGebra, se convierten en aliados para interpretar y visualizar conceptos matemáticos desde diversas perspectivas.

Por otra parte, La Plata (2014) realizó un análisis de los errores identificados con relación a la comprensión de la definición formal del límite de una función, los cuales fueron analizados mediante los criterios de Sierpinska (1987), así como de aspectos cognitivos mediante el enfoque ontosemiótico. Se concluyó que los conceptos previos de las funciones y los números reales son los que ocasionan una serie de obstáculos epistemológicos para la comprensión de los límites finitos para una función. Por tal motivo, en la educación media secundaria se debe tener en cuenta el contexto donde se realizan los procesos que normalmente son desarrollados en un espacio físico y que por motivos de la pandemia, se llevan a cabo mediante la implementación de herramientas y plataformas tecnológicas, con el objetivo de gestionar los aprendizajes, ya sea de manera sincrónica o asincrónica (Vásquez, Ruz y Martínez, 2020).

Ahora bien, diversas investigaciones se han realizado en tiempos de pandemia, que abarcan la enseñanza de los contenidos utilizando herramientas tecnológicas. Ejemplo de esto es la investigación realizada por Porlán (2020) que presenta una alternativa de un modelo de enseñanza-aprendizaje en tiempos del COVID-19, enfocada en irrumpir de manera impetuosa la modalidad virtual, indicando que es de vital importancia un cambio en la educación, que tenga como epicentro las nuevas Tecnologías de la Información y Comunicación (TIC). Todo esto, enmarcado en una problemática que afecta a la educación en general, la cual no está preparada para la inclusión de los usos tecnológicos, ni siquiera como atención a la diversidad, lo cual ha llevado a que el sector educativo utilice más estos recursos, dadas las condiciones de enseñanza de la actualidad.

Por supuesto que, existen algunas instituciones educativas que han tratado de realizar espacios de formación a profesores con respecto al tránsito de la presencialidad a la virtualidad, especialmente en países desarrollados. En países iberoamericanos es una constante la escasa preparación del profesorado para atender mediante recursos tecnológicos la enseñanza de las diversas áreas del conocimiento (Fardoun et al., 2020). Lo anterior se alinea con la investigación desarrollada por Palomo, Ruiz y Sánchez (2006), en la ciudad de Andalucía, España, la cual se titula: "Las TIC como agentes de innovación educativa". 
Estos autores manifiestan que las TIC facilitan las interacciones, que van desde una actitud más pasiva por parte de los estudiantes, a un comportamiento más equilibrado en la transformación de los procedimientos y los contenidos, por lo que la construcción del conocimiento es el resultado de interacciones sociales y del uso del lenguaje, donde el aprendizaje es más bien una experiencia compartida -social- que una experiencia individual (Calzadilla, 2002).

Por su parte, Rodríguez et al. (2014) muestran que el uso de la tecnología en los procesos de enseñanza de las matemáticas contribuye a que los estudiantes se sientan más cómodos y a gusto en el aprendizaje de contenidos históricamente enseñados de manera tradicional. Es por eso que dentro de las distintas adecuaciones que se han realizado al currículo de matemáticas, se sugiere que el uso de las calculadoras y computadores para los estudiantes son de gran apoyo para facilitar su proceso de aprendizaje. En concordancia con esto, Alpizar (2007), especifican que software como The Geometer's Sketchpad y Cabri Géomètre, facilitan la enseñanza de las matemáticas, ya que esta puede ser vista desde la óptica constructiva, geométrica y visual para algunos conceptos y definiciones. Es por esta razón que en la propuesta innovadora que se desarrolla en esta investigación, se utiliza el software especializado de las matemáticas GeoGebra, con el fin de facilitar el proceso de enseñanza y aprendizaje del límite de una función y sus propiedades.

\section{Marco teórico}

\section{Construcción histórica del concepto de límite}

La evolución del concepto de límite ha tenido muchas modificaciones en su comprensión geométrica, funcional, numérica, algebraica y topológica a lo largo de la historia de las matemáticas. De este modo, en este apartado se presenta cómo fue avanzando el desarrollo de este concepto y su construcción formal.

Durante las primeras apariciones intuitivas del concepto de límite, se comenzaron a realizar los primeros avances durante los años 400 A.C y 300 A.C. En ese entonces, era considerado simplemente un proceso implícito para darle soluciones a problemas contextuales como los de cálculo de área, velocidades y rectas tangentes a una curva. En los aportes realizadas por Trujillo, Vera y Saraza (2019) y Espíritu y Navarro (2015) se hizo una revisión de las aproximaciones al concepto de límite que realizaron autores clásicos, desde el punto de vista geométrico, Newton lo definió como "una cantidad (Cociente) a la cual una razón de cantidades en movimiento se aproxima continuamente, más que cualquier diferencia dada y no puede alcanzarla o sobrepasarla antes que las cantidades hayan decrecido indefinidamente"; Leibniz, por su parte, dio cuenta en la teoría de cálculo diferencial que "la pendiente de la tangente a una curva depende de la razón entre las diferencias de las orde- 
nadas y de las abscisas, cuando estas diferencias se hacen infinitamente pequeñas".

Sin embargo, es importante resaltar que antes de los aportes de Newton se encuentran los hallazgos realizados por Johannes Kepler (1571-1630), Bonaventura Cavalieri (1598-1647) y Pierre de Fermat (1601-1665), quienes luego de trabajar con figuras geométricas para hallar el área, el volumen o los extremos de una curva, introducen el concepto del análisis infinitesimal que posteriormente fue debatido por diferentes matemáticos. Además, según Bouazzaoui, Cornu y Deledicq (1994), este primer avance histórico hace referencia a la primera concepción que se tuvo del concepto de límite geométrico (Trujillo, Vera y Saraza, 2019).

De igual modo, el concepto formal del límite de una función, y sus más relevantes representaciones, son las realizadas desde el punto de vista geométrico, así como desde la forma analítica y numérica, lo que trae dificultades en los procesos de enseñanza - aprendizaje, de los límites, vistos en el sentido que se utiliza en la cotidianidad, así como en la forma en que se pretende generalizar las diferentes propiedades en los aspectos numerables y no numerables; junto a ello, otras dificultades que los estudiantes van presentando de acuerdo con la terminología y representaciones simbólicas como se presenta la definición formal del límite (Radillo y González, 2014).

Asimismo, el concepto del límite hace parte del análisis matemático, enmarcado en un nivel de pensamiento matemático superior, que implica que no sea comprendido con facilidad por los estudiantes, puesto que se requiere de un razonamiento riguroso y deductivo para su aprendizaje. Pese a esto, Radillo y González (2014) afirman que la complejidad de este concepto no es impedimento para que pueda ser trabajado por los profesores de matemáticas en la educación media. Los autores plantean que la enseñanza de este contenido en este nivel académico es una oportunidad para que los profesores puedan reflexionar acerca de su práctica e identificar las causas que conducen a la poca comprensión de los estudiantes acerca del tema, así como futuras propuestas para el abordaje efectivo de este contenido mediante otro tipo de recursos.

Por otro lado, Aquere et al (2007) muestra que en los procesos de la creación de distintos conceptos, como es el caso del límite de una función, es necesario tener una buena coordinación en los distintos registros que pueden representar un contenido, ya que esto genera mayor comprensión del contenido que se está enseñando. Algo en relación con lo afirmado por Volveras-Espinosa (2015), respecto a que las matemáticas no deben ser visualizadas solo como una reproducción maquinaria de algunas técnicas o formulas particulares y operativas, sino que también deberían ser vistas como una serie de codificación autónoma en la que los estudiantes puedan comprender diversas maneras de representar, entender y analizar una misma problemática. Un ejemplo de esto, es el caso del límite de una función, el cual puede ser estudiado no solamente de manera tradicional (definición por teoría), sino gráficamente y más si este tipo de registro se lleva a cabo mediante el uso de herramientas tecnológicas, como del registro numérico -haciendo uso de cálculos en tablas de valores-; de igual forma, mediante el registro verbal, comprendiendo el significado del límite en una función. 


\section{El Modelo de Van Hiele}

La importancia de la enseñanza y aprendizaje del límite de una función radica en que dentro del estudio del cálculo diferencial, los estudiantes suelen presentar algunas dificultades como lo son: la poca ejercitación en la realización de cálculos de tipo numérico y algebraico, los escasos conocimientos de relaciones entre los contenidos matemáticos con áreas afines como la química, física e ingeniería. Por ello, este trabajo se centra en la adquisición y comprensión del cálculo diferencial, especialmente el contenido del límite de una función de manera didáctica y dinámica. De esta manera, puede ser aplicado por los estudiantes en diversas situaciones del diario vivir (Artigue et al., 1991). De allí que esta investigación tenga en cuenta la adaptación a las fases propuestas por Van Hiele (1957), enfocadas en la enseñanza y aprendizaje de un contenido de las matemáticas y que se citan a continuación:

\section{Primera fase: Preguntas e información}

Esta se enmarca en identificar y determinar cómo pueden acercarse los estudiantes a una situación real contextualizada. Para cumplir una de las frases más célebres de Ausubel: Averígüese esto y enséñese en consecuencia. En esta fase se desarrolla la expresión oral del estudiante mediante la realización de una serie de preguntas encaminadas a encontrar el inicio de conocimiento por parte de los estudiantes acerca del tema de estudio y poder seguir el proceso.

\section{Segunda fase: Orientación dirigida}

En esta fase, se tiene en cuenta la capacidad didáctica y dinámica del profesor para identificar el rendimiento de cada uno de los estudiantes acerca del contenido objeto de estudio, y a partir de eso, obtener resultados óptimos en el tiempo estipula- do durante el desarrollo de la propuesta pedagógica. Específicamente que el estudiante sea capaz de comprender, descubrir, asimilar y aplicar todos los conocimientos adquiridos en relación al límite de una función y sus propiedades.

\section{Tercera fase: Explicación y explicitación}

En esta frase se producen una serie de intercambios de experiencias e ideas entre los estudiantes; el rol del docente gira en torno a ser un guía y revisor de alguno de los posibles errores que puedan cometer los estudiantes en el intercambio de conocimientos relacionados con el límite de funciones y sus propiedades.

\section{Metodología}

Esta investigación se desarrolla bajo un enfoque cualitativo, ya que se basa principalmente en la percepción y comprensión humana, de manera que a partir de la interpretación, descripción y comprensión de los fenómenos, se establezcan percepciones y significados producidos por las experiencias de los participantes (Stake, 2010). El diseño de la investigación es de tipo acción participativa (Hernández-Sampieri, 2014), en tanto su característica principal radica en el cambio de una realidad, lo cual debe incorporarse en el propio proceso de investigación, en donde se indaga al mismo tiempo que se interviene, es decir la investigación se realiza a partir de la interacción: investigador-participantes. En este contexto la población tiene una participación muy activa con el agente investigativo, ya que para poder realizar un análisis de la realidad, a partir de las dificultades que se encuentran en el aprendizaje de los estudiantes acerca de la definición de límite formal, provocado por la enseñanza de manera tradicional en tiempos de pandemia, mediante plataformas virtuales y uti- 
lizando presentación de diapositivas en Power Point como reemplazo al tablero. Se propone una unidad didáctica utilizando el software especializado de las matemáticas GeoGebra, como herramienta de mediación para la enseñanza de este contenido en estudiantes de undécimo grado de una institución educativa de la ciudad de Barranquilla.

Las fases de la investigación, se adaptan del trabajo realizado por Conde-Carmona y Fontalvo (2019); en primer lugar, con la formulación se explica y precisa el qué y el cómo se va investigar. Así, mediante una serie de observaciones se evidencia que los estudiantes presentaban dificultades para comprender el concepto de límite de una función de manera formal; luego se lleva a cabo a partir de lo observado, el diseño, que responde a la pregunta de investigación sobre la manera en que se llevará a cabo el estudio y sobre la ubicación espacio-temporal. Por último, en la ejecución se definen las técnicas que permitan el contacto con el sujeto, tales como la entrevista y la elaboración colectiva de actividades y talleres (unidad didáctica) para la enseñanza del concepto de límite de una función mediante GeoGebra.

\section{Participantes}

La población de esta investigación está establecida en los grados once del Colegio San José Hermanitas de la Anunciación, con 52 estudiantes que los conforman, ubicado en el suroriente de Barranquilla. Esta institución en las pruebas ICFES posee un nivel básico en las competencias de matemáticas. Para la selección de la muestra se tomó el curso de once grado A, conformado por 20 estudiantes de una edad que oscila entre 15 y 17 años de sexo masculino y femenino. Esta selección se realizó por las dificultades evidenciadas en el aprendizaje de las matemáticas de los estudiantes durante el I y II periodo, mediante un muestreo no probabilístico que según Hernández-Sampieri (2014) se desarrolla cuando la escogencia de las opciones no dependen directamente de la probabilidad, si no que van ligadas directamente al investigador, o a la población, desde la que se escoge la muestra.

\section{Técnicas e instrumentos de recolección de información}

\section{La Observación no participantes en clases remotas}

Esta técnica fue aplicada a los estudiantes durante las distintas actividades programadas en línea, con el fin de evidenciar la actitud que tienen frente al tema, y el interés que presentan ambos grupos de acuerdo con el tipo de enseñanza. En esta técnica el investigador asume un rol pasivo, tan solo de recopilar datos, sin interferir en el normal comportamiento de lo que está investigando (Ynoub, 2015). El instrumento que se usó para recolectar la información a partir de ésta técnica fue el registro anecdótico el cual se realizó al momento de finalización de la propuesta.

\section{La Entrevista con videollamada por Meet}

Esta técnica se fundamenta directamente con el individuo (Herrera, 2017), que en esta investigación hace referencia al paradigma utilizado en este trabajo de transformación social Esta técnica fue aplicada al profesor de matemáticas de grado 11A. Se le realizó una serie de preguntas previamente establecidas, relacionadas con la metodología usada para la enseñanza del límite de una función y su demostración formal en grado $11 \mathrm{~A}$, con el fin de evidenciar la forma en que les facilita el aprendizaje a los estudiantes. El instrumento usado fue una guía de 
entrevista estructurada, el cual fue realizado antes de la implementación.

\section{La Encuesta con google}

Esta técnica es una serie de procesos o procedimientos, que facilita la indagación sobre asuntos que están ligados al sujeto; a través de esta se pueden adquirir los datos exactos sobre un número considerable de personas (Grasso, 2006). Esta técnica fue aplicada a los estudiantes de grado $11 \mathrm{~A}$, mediante un cuestionario (examen diagnóstico), conformado por una serie de preguntas sobre los saberes previos del límite de una función y el aprendizaje de este concepto, con el fin de obtener información individual y grupal sobre la situación problema.

\section{Validez}

Para medir la validez del cuestionario aplicado a los estudiantes, este fue sometido a un juicio de $3 \mathrm{ex}-$ pertos, quienes agregaron o quitaron preguntas, dependiendo de si los ítems guardaban o no relación con la variable objeto de estudio. Esto se realizó para la prueba diagnóstica en línea, que inicialmente constaba de 12 preguntas, pero luego de estas revisiones quedó reducida a 9 preguntas, las cuales, ya validadas, fueron puestas a disposición del objeto de estudio para seguir en marcha la investigación (Suarez, 2015).

\section{Propuesta pedagógica}

Con el objetivo de brindar una mejor manera de afrontar los desafíos provocados por la pandemia, se plantea una propuesta que interviene en el contexto actual de la educación, la cual lleva como título: "Las TIC como propuesta dinámica, para el fortalecimiento de competencias matemática, usando como objeto matemático el concepto de límite en tiempos de pandemia”. Esta propuesta pedagógica se desarrolló mediante una serie de actividades, las cuales fueron presentadas en clases virtuales, pese a la contingencia ocasionada por el COVID-19. Así mismo, esta propuesta tuvo como actividad inicial una prueba diagnóstica secuencial, con el fin de caracterizar los saberes previos de los estudiantes para que a partir de allí, se desarrollarán actividades relacionadas con el límite de una función, sus propiedades y aplicaciones, además de diversas formas de concretar la abstracción de este concepto de forma dinámica mediante el software GeoGebra, lo que a su vez permitiría que todos los estudiantes se involucraran en la actividades.

Con respecto a lo planteado anteriormente, se realizaron todos esos procedimientos siguiendo las fases de Van Hiele (1957); ya en la virtualidad se hace útil la visualización de los procesos matemáticos como los que plantea este autor: observación, análisis, deducción formal e informal en la enseñanza del límite de una función de manera gráfica mediante GeoGebra. Además, esta propuesta es de vital importancia porque permite encontrar las distintas formas de concretar el aprendizaje del límite de una función en tiempos de pandemia, de tal manera que facilite el proceso de enseñanza-aprendizaje.

Por otro lado, Bravo y Arrieta (2003) afirman que la demostración de alguna afirmación matemática ayuda de manera directa a la buena interconexión de los conceptos o saberes previos para solucionar problemas contextuales, o bien, a algunas operaciones concretas en donde sea necesario analizar, comparar, sintetizar, particularizar, clasificar y analizar que los trabajos que requieren demostrar teoremas matemáticos, tienen una vital importancia en el desarrollo de destrezas que ayudan en el pensamien- 
to matemático de los estudiantes. De esta manera, es relevante la implementación de esta propuesta, porque permite presentar la definición del límite de una forma dinámica y didáctica en estos tiempos de pandemia, además, que el proceso de enseñanza esté más acorde a las necesidades del contexto educativo actual; así mismo, permite desarrollar en el estudiante una mejor forma de analizar e interpretar las distintas demostraciones del límite de una función y las propiedades de estos.

En línea con lo anterior, la incorporación de las TIC en las instituciones educativas se ha realizado con el fin de tener una mejora en cada uno de los procesos de enseñanza y aprendizaje, así como en el comportamiento de la gestión educativa (MEN, 2003), lo que muestra que los entes nacionales ven con buenos ojos el uso de estas herramientas con el fin de mejorar la calidad educativa. Así, el objetivo de esta propuesta consistió en fortalecer los procesos de enseñanza y aprendizaje colaborativo en tiempos de pandemia del límite de una función mediado por las TIC de manera dinámica. En la tabla 1 se muestra el plan de acción que siguió la propuesta pedagógica implementada con estudiantes de undécimo grado de una institución educativa de la ciudad de Barranquilla con la respectiva descripción de las actividades.

Tabla 1. Plan de acción de la propuesta pedagógica.

\begin{tabular}{|c|c|c|c|c|}
\hline $\begin{array}{c}\text { Eventos } \\
\text { pedagógicos }\end{array}$ & Meta & Recursos & Tiempo & Evaluación \\
\hline $\begin{array}{l}\text { Actividad 1: } \\
\text { Presentación } \\
\text { del límite de } \\
\text { una función; } \\
\text { demostraciones y } \\
\text { propiedades. }\end{array}$ & $\begin{array}{l}\text { Que los educandos } \\
\text { visualicen y } \\
\text { comprendan las } \\
\text { propiedades del } \\
\text { límite de una } \\
\text { función y su } \\
\text { demostración, y } \\
\text { lo relacionen con } \\
\text { elementos de su } \\
\text { contexto. }\end{array}$ & \multirow{2}{*}{$\begin{array}{c}\text { PC y Google Meet. } \\
\text { Tableros digitales. } \\
\text { GeoGebra. }\end{array}$} & 5 - 4 horas. & $\begin{array}{c}\text { Talleres grupales } \\
\text { sobre situaciones } \\
\text { problemas del } \\
\text { límite de una } \\
\text { función y sus } \\
\text { propiedades. }\end{array}$ \\
\hline $\begin{array}{l}\text { Actividad 2: Análisis } \\
\text { de las aplicaciones } \\
\text { de límites } \\
\text { especiales con las } \\
\text { TIC; estrategias } \\
\text { dinámicas. }\end{array}$ & $\begin{array}{l}\text { Que los alumnos } \\
\text { puedan analizar y } \\
\text { reflexionar sobre } \\
\text { las aplicaciones } \\
\text { del límite de una } \\
\text { función por medio } \\
\text { de las TIC y la } \\
\text { simulación. }\end{array}$ & & 3 horas. & $\begin{array}{c}\text { Evaluación } \\
\text { colaborativa. }\end{array}$ \\
\hline
\end{tabular}


Continuación Tabla 1. Plan de acción de la propuesta pedagógica.

\begin{tabular}{|c|c|c|c|c|}
\hline $\begin{array}{c}\text { Eventos } \\
\text { pedagógicos }\end{array}$ & Meta & Recursos & Tiempo & Evaluación \\
\hline $\begin{array}{l}\text { Actividad 3: } \\
\text { trabajos grupales } \\
\text { con el fin de } \\
\text { que se realicen } \\
\text { intercambio de } \\
\text { ideas sobre el límite } \\
\text { de una función y } \\
\text { las propiedades } \\
\text { de estos en casos } \\
\text { especiales entre } \\
\text { estudiantes, de } \\
\text { manera lógica, } \\
\text { intercambiar } \\
\text { correcciones en el } \\
\text { uso del lenguaje } \\
\text { matemático. }\end{array}$ & $\begin{array}{l}\text { Que los estudiantes } \\
\text { describan las } \\
\text { propiedades de los } \\
\text { límites especiales } \\
\text { de manera formal } \\
\text { y que identifiquen } \\
\text { situaciones } \\
\text { problemáticas } \\
\text { donde no haya } \\
\text { una solución de } \\
\text { manera inmediata y } \\
\text { aplicarlos. }\end{array}$ & $\begin{array}{c}\text { PC y Google Meet. } \\
\text { Tableros digitales. } \\
\text { GeoGebra. }\end{array}$ & 3 horas. & $\begin{array}{l}\text { Una autoevaluación } \\
\text { de todo el proceso } \\
\text { desarrollado. }\end{array}$ \\
\hline
\end{tabular}

Fuente: Elaboración propia.

Cabe destacar, que los aspectos pedagógicos de esta propuesta se basaron en los niveles de aprendizaje de Van Hiele; en este caso la investigación se limitó a los tres primeros niveles que están ligados por completo a las tres fases que plantea dicho teórico. A pesar de ser 5 niveles y asimismo 5 fases, sólo se muestran estos porque el autor manifiesta en su teoría que estas fases pueden ser desarrolladas a nivel de secundaria (Van Hiele, 1957); también porque este trabajo se interesó en desarrollar esas 3 fases.
Como primer punto en esta propuesta, se realizó, una visualización o reconocimiento de la definición de límite de una función, en donde se observan los objetos de una manera concreta y total, sin que exista alguna distinción de sus características y mecanismos. La idea de esta actividad consistía en que los estudiantes encontraran una relación con su contexto a partir de lo representado en la Figura 1, que hace alusión a la representación de la definición de límite plasmado mediante una función cualquiera en GeoGebra. 


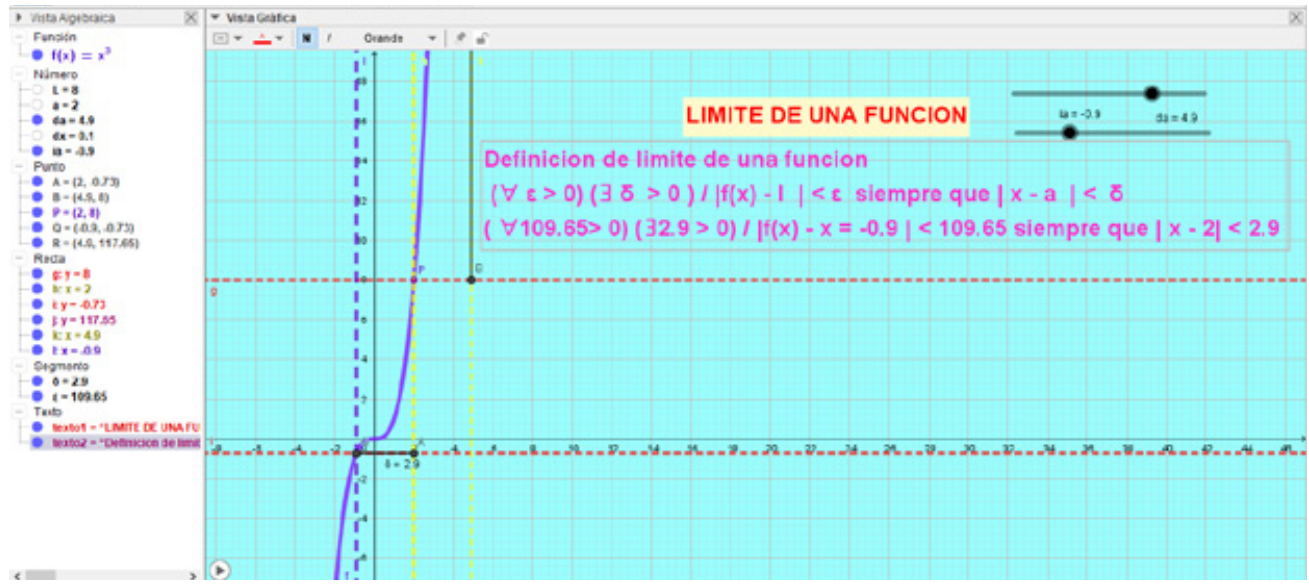

Figura 1. Representación de la definición de límite de una función en GeoGebra.

Fuente: Elaboración propia.

Posterior a esto, se realizaron ejercitaciones con el fin de que los estudiantes realizaran análisis de la representación gráfica de la definición de límite y percibieran que las características y propiedades de las figuras y cuerpos a partir de la observación y la experimentación se dan de manera informal, y permiten describir las características principales del límite de una función como se observa en la figura 2.

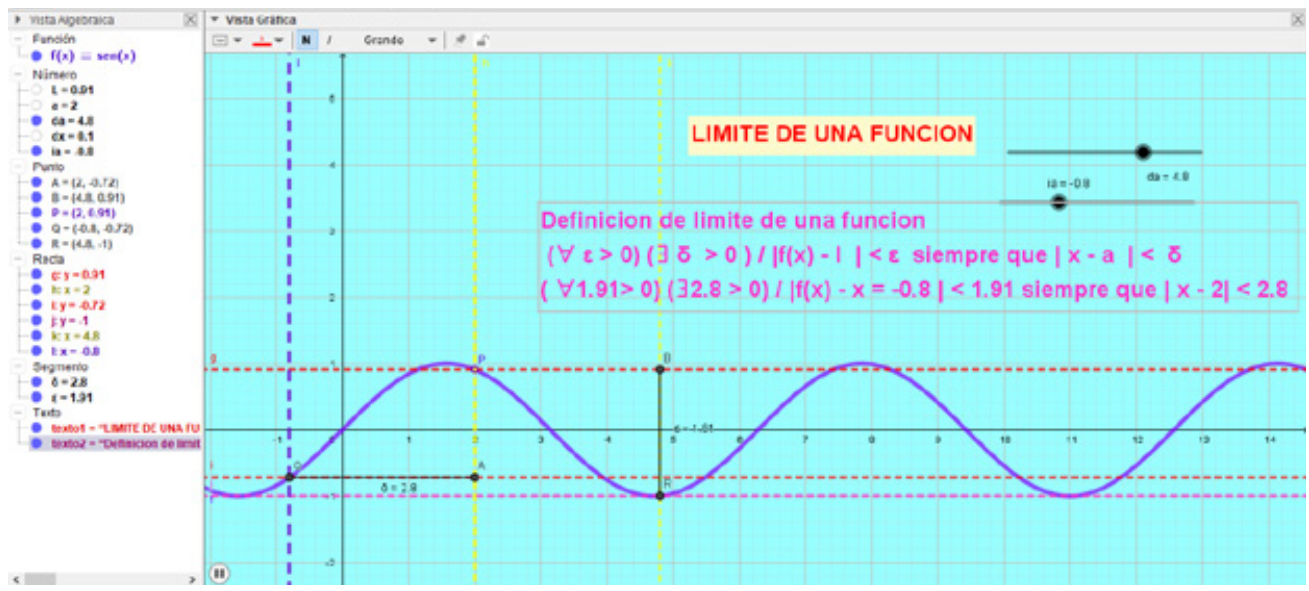

Figura 2. Representación de las características del límite de una función en GeoGebra.

Fuente: Elaboración propia. 
Un tercer acto pedagógico, se llevó a cabo con la ayuda de las anteriores actividades (Figuras 1 y 2). Se hizo una clasificación de las figuras formalmente, es decir se mencionaron los componentes necesarios y suficientes que deben tener, lo cual es de vital importancia ya que permite comprender el verdadero significado de la abstracción de las definiciones que se desarrollan en las clases de matemáticas. A su vez, contribuye en la realización de clasificaciones lógicas de manera más rigurosa, debido a su avance en el razonamiento matemático, pues permite que se produzcan intercambios de pensamiento de ideas de manera organizada, para que se produzca un aprendizaje colaborativo entre los estudiantes.

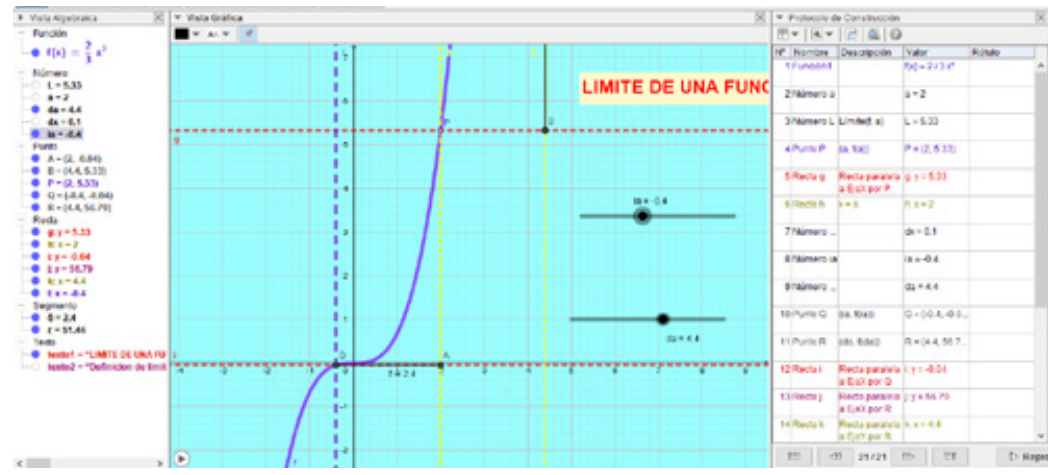

Figura 3. Ejemplificación de la definición de límite de una función gráficamente en la función $f(x)=x^{\wedge 3}$.

Fuente: Elaboración propia.

Por otro lado, dentro de esta propuesta de intervención se tuvo en cuenta la evaluación del proceso, la cual se concibe como el conjunto de procesos de forma continua entre la enseñanza-aprendizaje del límite de una función en tiempos de COVID-19, así como la evolución del aprendizaje real para poder mejorar y afinar la intervención pedagógica (Porlán, 2020). De igual manera, la autoevaluación de la propuesta es un mecanismo que permite analizar los resultados obtenidos por los estudiantes durante la aplicación de la propuesta, reflexionar y criticar sobre estos, y a su vez, construir planes de mejora para el desarrollo de las actividades (Calatayud, 2002). El instrumento utilizado para realizar la autoevaluación fue un cuestionario con preguntas abiertas que le permitiera al estudiante hacer una evaluación de su proceso, con respecto al aprendizaje del límite de una función y sus propiedades. Ahora bien, debido a que estos espacios de aprendizajes colaborativos se desarrollaron de manera grupal mediante debates abiertos con una intención dirigida, es significativo resaltar que permitieron mostrarle a los estudiantes el compromiso que se requiere para el aprendizaje de las matemáticas, especialmente si se trata de contenidos complejos como lo es el límite de funciones y sus propiedades (Lillo, 2012).

\section{Discusión de los resultados}

A continuación, se muestra la discusión de los resultados obtenidos a partir de los instrumentos aplicados en la presente investigación: 
Tabla 2. Análisis de la prueba diagnóstica.

\begin{tabular}{|c|c|}
\hline Preguntas & Análisis e interpretación \\
\hline $\begin{array}{l}\text { Explique sus ideas } \\
\text { o concepciones } \\
\text { acerca del límite de } \\
\text { una función. }\end{array}$ & $\begin{array}{l}\text { Luego de realizar el análisis de las respuestas dadas por los estudiantes a esta pregunta, se } \\
\text { evidenció que el } 88 \% \text { de los estudiantes conocen los términos del límite de una función. Sin } \\
\text { embargo, la mayoría la mayoría afirmó que el límite de una función se aplica en funciones } \\
\text { pero agregan que no saben la utilidad de este concepto en ninguna situación del diario vivir, } \\
\text { lo que evidencia que el límite de una función es conocido por los estudiantes solamente } \\
\text { en su presentación simbólica, más no en su aplicación en diversas áreas afines a las } \\
\text { matemáticas. }\end{array}$ \\
\hline $\begin{array}{l}\text { Haga un esquema } \\
\text { que relacione } \\
\text { la definición o } \\
\text { demostración } \\
\text { que conoce sobre } \\
\text { el límite de una } \\
\text { función. }\end{array}$ & $\begin{array}{l}\text { Se evidenció que un alto porcentaje de los estudiantes no realizaron de manera correcta } \\
\text { la representación geométrica del límite de una función, escasamente el } 11,5 \% \text { de los } \\
\text { estudiantes contestaron bien esta pregunta, sin dejar a un lado los } 4 \text { estudiantes que } \\
\text { equivalen al } 15,3 \% \text { de la muestra que no contestaron esta pregunta. Con relación a estas } \\
\text { respuestas expresadas por los estudiantes, queda en manifiesto que presentan debilidades } \\
\text { en cuanto a la demostración geométrica de la definición del límite de una función. }\end{array}$ \\
\hline $\begin{array}{l}\text { Explique las } \\
\text { dificultades que } \\
\text { ha tenido cuando } \\
\text { le han explicado } \\
\text { el límite de una } \\
\text { función. }\end{array}$ & $\begin{array}{l}\text { A partir de las respuestas dadas se puede inferir que los estudiantes no tienen claro la } \\
\text { definición del límite de una función, ya que el } 76,9 \% \text { contestaron a esta pregunta que } \\
\text { no entendieron nada sobre estas temáticas. Así mismo, aseguraron que no sabían que } \\
\text { significaban palabras como épsilon y delta, claves en la definición formal de límite y que } \\
\text { tampoco entendían la relación y el acercamiento que tienen estas dos variables en la } \\
\text { función, así como tampoco el discurso que proponía el profesor para la enseñanza de este } \\
\text { contenido. Es interesante que los estudiantes manifiestan que no veían en la realidad lo que } \\
\text { el profesor les explicaba, consideran que el tablero no era el mejor medio para explicar un } \\
\text { contenido complejo como el del límite. Por otra parte, los estudiantes manifestaron que no } \\
\text { entendían los límites infinitos y sus propiedades. } \\
\text { De acuerdo con estos resultados obtenidos, se pone en evidencia que los procesos de } \\
\text { enseñanza pese a la virtualidad, siguen siendo mediante del tablero, con la única variable } \\
\text { que este es de tipo digital, lo cual ocasiona que los estudiantes presenten este tipo de } \\
\text { respuesta, mostrando sus dificultades en el proceso de aprendizaje. Cabe resaltar que los } \\
\text { estudiantes que afirmaron no presentar dificultad en la explicación del límite de una función, } \\
\text { no tuvieron buenos resultados en la solución de los problemas planteados en la prueba. }\end{array}$ \\
\hline
\end{tabular}

Continúa 
Continuación Tabla 2. Análisis de la prueba diagnóstica.

\begin{tabular}{|c|c|}
\hline \multicolumn{2}{|c|}{ Preguntas } \\
\hline \multicolumn{2}{|c|}{$\begin{array}{l}\text { A qué tiende el } \\
\text { siguiente límite }\end{array}$} \\
\hline $\lim$ & $x^{2}-4$ \\
\hline$x \rightarrow 2$ & $x-2$ \\
\hline \multicolumn{2}{|c|}{$\begin{array}{l}\text { Explique su } \\
\text { respuesta de } \\
\text { acuerdo con lo } \\
\text { aprendido en clases }\end{array}$} \\
\hline \multicolumn{2}{|c|}{$\begin{array}{l}\text { Cuándo les } \\
\text { presentaron la } \\
\text { definición del límite } \\
\text { de una función y su } \\
\text { demostración, ¿lo } \\
\text { hicieron con ayuda } \\
\text { de la tecnología? } \\
\text { Explique. }\end{array}$} \\
\hline
\end{tabular}

¿Conoce algún software para trabajar el límite de una función?
Se observó en las soluciones que los estudiantes poseen dificultades para realizar la diferencia de cuadrados y las condiciones que se deben tener en cuenta al momento de reemplazar el límite de manera inmediata. En cuanto a las de realizar cancelaciones de términos semejantes, lo cual es relacionado con las respuestas anteriores, manifestaron de forma escrita que presentaban dificultades en la comprensión de la definición del límite de una función, porque si no se entiende la conceptualización, mucho menos se comprenderá su desarrollo.
En las respuestas de los estudiantes se encontró una contradicción puesto que algunos afirmaron usar las TIC en los procesos de enseñanza - aprendizaje del límite de una función, mientras que otros decían lo contrario; se dice que es una contradicción porque ambos grupos asistieron a la misma clase, dictada por el mismo profesor. A partir de esto, se pudo concluir que, si están en lo correcto los estudiantes que dicen que sí, entonces la clase no fue significativa para los estudiantes que afirman que su profesor no utilizó la tecnología para la enseñanza de este contenido. Sin embargo, es importante resaltar que entre más herramientas tecnológicas utilice el profesor para la enseñanza de un contenido de las matemáticas, mayores oportunidades tienen los estudiantes para explorar estos contenidos desde diversas perspectivas. No obstante, a muchos profesores les da temor explorar programas matemáticos distintos a los que ya conocen desde hace años, sobre todo porque es posible que algún alumno lo deje en evidencia si le pregunta algo que no sabe (Fontal, Marín y García, 2015). De modo que la educación tradicional continúa en las aulas por razones como las antes mencionadas.
En este apartado se encontró que la mayoría de los estudiantes no conoce un software para trabajar el límite de una función, por lo que se puede deducir que, para la enseñanza de este, el profesor no recurrió a un programa o software que le permitiera al estudiante observar y analizar de manera dinámica el límite de una función, así como su demostración para poder comprenderlo de mejor manera y aplicarlo además en diversas situaciones de la vida cotidiana o de áreas afines a las matemáticas.

Fuente: Elaboración propia. 
Tabla 3. Análisis de la entrevista y del registro anecdótico.

\section{Análisis de la entrevista}

De acuerdo con las respuestas dadas por la profesora en la entrevista, se puede afirmar que esta no presenta las temáticas a los estudiantes con ayuda de las TIC, específicamente mediante software especializado del área que faciliten el proceso de enseñanza-aprendizaje. Además, se encontró que en el colegio no se hacen capacitaciones a los profesores en cuanto al uso de las TIC con sentido pedagógico, debido a que la docente en una de las respuestas afirmó que tiene el deseo de mostrar a los estudiantes las clases con ayuda de la tecnología, pero no sabe cómo proceder debido a su escaso conocimiento y grado de aplicación en el aula para la enseñanza de contenidos de las matemáticas.

Además, señaló que no hacía mucha reflexión de sus prácticas, y que replicaba prácticas aprendidas de sus maestros, siendo esto un hecho no favorable para los procesos de enseñanza - aprendizaje actuales, debido que la sociedad actual exige que el docente reflexione constantemente sus prácticas, para poder hacer una evaluación de las mismas y así mejorar los procesos (Valbuena, Conde y Ortiz, 2018; Valbuena, Conde-Carmona y Padilla-Escorcia, 2018). No obstante, los docentes siguen manteniendo en su quehacer pedagógico, métodos de enseñanza tradicionales, pese a que la educación está en una transición de la presencialidad a la virtualidad, dada la contingencia ocasionada por la pandemia de COVID -19., lo cual se desalinea con lo propuesto por Cardozo, Duarte y Fernández (2021) con respecto a que las necesidades en el contexto educativo actuales implica en los profesores una motivación distinta a la tradicional, puesto que se pretende que los estudiantes construyan su propio conocimiento.

En ese sentido lo afirmado por la profesora, afecta de forma directa al estudiantado; un ejemplo de esto son los resultados obtenidos en la prueba diagnóstica. Por lo tanto, es prudente afirmar que la falta de motivación en los estudiantes, más allá de que los profesores utilicen o no recursos tecnológicos como mediadores en los contenidos, debe ser urgentemente atendido en las escuelas, y más si se tiene en cuenta las modalidades de enseñanza que emergen en el país hoy día.

\section{Análisis del registro anecdótico}

En los resultados del registro anecdótico se evidenció una transformación en el grado undécimo, partiendo de la realización de la prueba diagnóstica en la que se identificaron las principales dificultades que presentaban los estudiantes con respecto al límite de una función y sus propiedades. Dentro de estas se resaltaba el escaso conocimiento y comprensión de nociones, definiciones y propiedades del límite de una función.

En ese orden, se observó cambios significativos en la actitud de los estudiantes, quienes habían afirmado que su profesora no hacía uso de software matemáticos dinámicos para la enseñanza de las matemática, y que incluso en la virtualidad utilizaban el tablero para explicar los contenidos, solamente que de manera digital, ya que después del desarrollo de la propuesta afirmaron que se sintieron más motivados frente a la temática tratada, ya que la implementación de las TIC para el aprendizaje del límite de una función y sus propiedades mediante el uso del software GeoGebra, permitió mayor comprensión acerca de la demostración geométrica del límite de una función, como se observa en las figuras 1 y 3.

Todo esto, a su vez está relacionado con lo propuesto por Palomo, Ruiz y Sánchez (2006) con respecto a que las TIC brindan una interconexión que hace que el estudiante pase de

Continúa 
Continuación Tabla 3. Análisis de la entrevista y del registro anecdótico.

\section{Análisis del registro anecdótico}

una actitud pasiva a una actitud más activa que busca la trasfiguración de los contenidos y procedimientos tradicionales.

Ahora bien, se observó que durante la mesa redonda, cuando los estudiantes daban su opinión acerca del proceso de implementación de las TIC para el aprendizaje del límite de una función y sus propiedades, se notó el dominio que tenían del tema al expresarse con seguridad. Además, se evidenció el manejo en la terminología formal utilizada en los intercambio de ideas, donde mencionaban palabras que ellos en la prueba diagnóstica decían que no sabían, como "épsilon y delta". Así mismo, se notaba la seguridad en el aprendizaje colaborativo, puesto que entre pares académicos se complementaban las ideas.

Fuente: Elaboración propia.

\section{Conclusiones}

De acuerdo con la pregunta problema planteada al inicio de este trabajo investigativo, se puede decir que la propuesta pedagógica: "Las TIC como propuesta dinámica para el fortalecimiento de competencias matemática, usando como objeto matemático el concepto de límite" suministró a los estudiantes una mayor apropiación del concepto de límite de una función y sus propiedades. Esto gracias a que permitió establecer relaciones en los estudiantes entre la abstracción del contenido con sus respectivas aplicaciones en la vida cotidiana. Así mismo, el desarrollo de trabajo colaborativo entre pares, contribuyó a que se diera más fácil la interpretación y aplicación de la definición de límite de una función y sus propiedades en GeoGebra, debido a la versatilidad que ofrecen este tipo de herramientas tecnológicas.

Además, la implementación de la propuesta aportó en la caracterización de los saberes previos que requieren los estudiantes para el aprendizaje del límite de una función, lo cual es de vital importancia para definir estrategias metodológicas que faciliten el aprendizaje de este concepto, y que se evidenció en los análisis realizados al diagnóstico y desarrollo de las actividades de la implementación de la propuesta aplicados a los estudiantes.

Por otro lado, a partir de las evaluaciones presentadas al finalizar la propuesta, se propició un aprendizaje colaborativo en el grupo de estudiantes, uno de los objetivos de la propuesta, lo que evidencia un avance significativo en la comprensión del límite de una función, su representación gráfica y propiedades, con respecto a la prueba diagnóstica aplicada a estos mismos estudiantes antes de implementar la propuesta.

Para concluir, cabe destacar que los retos presentados en la enseñanza de las matemáticas en tiempos de pandemia, fueron superados con una buena disposición por parte de los participantes. Dentro de estos, se destaca la organización y estructuración en el desarrollo de la propuesta, y que es muestra del desarrollo de competencias como la interpretación y representación de los contenidos matemáti- 
cos. Sin embargo, llama la atención que el desarrollo de estas competencias surgió por la necesidad que implicó la enseñanza de estos contenidos abstractos de las matemáticas con innovación y mayor interacción entre el estudiante y el profesor. Pese a esto, no deja de ser imperativo que los profesores que enseñan matemáticas en educación media o a nivel superior, tengan conocimiento de recursos tecnológicos especializados de la matemática que ayuden en la mediación de los contenidos que enseñan, de manera que los contenidos con nivel de abstracción alto puedan ser explorados de manera dinámica por los estudiantes, y que en ambientes distintos al aula presencial, también se desarrollen competencias del pensamiento matemático. 


\section{Referencias}

Aldon, G., Cusi, A., Schacht, F., y Swidan, O. (2021). Teaching Mathematics in a Context of Lockdown: A Study Focused on Teachers' Praxeologies. Education Sciences, v.11, n. 38, pp. 1-21. https://doi. org/10.3390/educsci11020038

Alpizar, M. (2007). Herramientas tecnológicas en el proceso de enseñanza y aprendizaje de la estadística. Cuadernos, pp. 96-115.

Aquere, S., Engler, A., Vrancken, S., Hecklein, M., Müller, D., y Gregorini, M. I. (2007). Nos preparamos para el cálculo trabajando sobre la recta real. Revista Premisa, v.9, n. 32, pp. 24-36.

Artigue, M., Douady, R., Moreno, L., y Gómez, P. (1995). Ingeniería didáctica en educación matemática un esquema para la investigación y la innovación en la enseñanza y el aprendizaje de las matemáticas. Grupo Editorial Iberoamérica.

Aznar-Sala, F. (2020). La Educación Secundaria en España en Medio de la Crisis del COVID-19, International Journal of Sociology of Education, pp. 53-78. http://doi.org/10.17583/rise.2020.5749

Bravo, L., y Arrieta, J. (2003). Una estrategia didactica para la enseñanza de las demostraciones en geometricas:resultado de su implementacion. En Castro, Encarnación (Ed.). Investigación en educación matemática : séptimo Simposio de la Sociedad Española de Investigación en Educación Matemática Granada: Universidad de Granada. pp. 153-160.
Calvo-Cereijo, M. (2019). Pensamiento complejo y transdisciplina. Sophia. Colección de Filosofía de la Educación, n. 26, pp. 307-326. https://.doi. org/10.17163/soph.n26.2019.09

Calzadilla, M. (2002). Aprendizaje colaborativo y tecnologías de la información y la comunicación. OEI-Revista Iberoamericana de Educación, v.29, n.1, pp. 1-10. https://doi.org/10.35362/ rie2912868

Cardozo, R., Duarte, J., y Fernández, F. (2021). Estrategia didáctica, mediada por TIC, para mejorar las competencias lectoescrituras en estudiantes de primero de primaria. Saber, Ciencia y Libertad, v.13, n. 2, pp. 235-247. https://doi.org/10.18041/23823240/saber.2018v13n2.4638

Cassibba, R., Ferrarello, D., Mammana, M., Musso, P., Penissi, M., y Taranto, E. (2021). Teaching Mathematics at Distance: A Challenge for Universities. Education Science, v. 11, n. 1, pp. 1-20. https://doi.org/10.3390/educsci11010001

Conde-Carmona, R, y Fontalvo, A. (2019). Didáctica del teorema de Pitágoras mediada por las TIC: el caso de una clase de Matemáticas. Trilogía, v. 11, n. 21, pp. 255-281. https://doi. org/10.22430/21457778.1187

Conde-Carmona, R., y Padilla-Escorcia, I. (2021). Aprender matemáticas en tiempos del COVID-19: un estudio de caso con estudiantes universitarios. Educación y Humanismo, v. 23, n. 40, pp. 1-19. https://doi.org/10.17081/eduhum.23.40.4380 
D’Amore, B. (2004). Conceptualización, registros de representaciones semióticas y noética: interacciones constructivistas en el aprendizaje de los conceptos matemáticos e hipótesis sobre algunos factores que inhiben la devolución. Revista Científica, n. 11, pp. 150-164. https://doi. org/10.14483/23448350.419

Departamento Nacional de Planeación. (2020). Documento Conpes 3988. https://www.dnp.gov. co/CONPES/documentos-conpes/Paginas/documentos-conpes.aspx

Duval, R. (1995). Sémiosis et pensée humaine. Registres sémiotiques et apprentissages intellectuels. Berne, Peter Lang, pp. 29-31

Espíritu, M. y Navarro, C. (2015). Límites indeterminados mediante el uso de tablas de valores y gráficas. Números, n. 88, pp. 31-53.

Failache, E., Katzkowicz, N., y Machado, A. (2020). Consecuencias del Cierre de Escuelas por el COVID-19 en las Desigualdades Educativas. Revista Internacional de la Educación para la Justicia Social, n. 9, pp. 1-9.

Fardoun, H., González, C., Collazos, C., y Yousef, M. (2020). Estudio exploratorio en Iberoamérica sobre procesos de enseñanza-aprendizaje y propuesta de evaluación en tiempos de pandemia. Education in the Knowledge Society, n. 21, pp. 1-9. https://doi.org/10.14201/eks.23537
Fontal, O., Marín, S., y García, S. (2015). Educación de las artes visuales y plásticas en educación primaria. Colección: Didáctica y Desarrollo. Ediciones Paraninfo, SA, pp. 240-242.

Granados-Ortiz, C., y Padilla-Escorcia, I. (2021). El aprendizaje gráfico de la recta tangente a través de la modelación de las secciones cónicas utilizando GeoGebra. Revista Científica, v. 40, n.1, pp. 118132. https://doi.org/10.14483/23448350.16137

Grasso. (2006). Encuestas: elementos para su diseño y analisis. Editorial Brujas. Cordoba- Argentina. pp. 184.

Guerrero, J., Hernández, L. (2020). Análisis de actividades didácticas para el estudio del límite de una función por medio de la teoría APOE. Investigación e Innovación en Matemática Educativa, v. 5, pp. 1-19. https://doi.org/10.46618/iime.70

Hernández-Sampieri, R. (2014). Metodología de la Investigación. McGRAW-HILL / Interamericana

Herrera. (2017). La investigación cualitativa. DSpace Repository, http://148.202.167.116:8080/ xmlui/handle/123456789/1167

Jiménez Galán, Y. I. (2017). Innovación educativa y docencia ifalla el protagonista?: el caso ESCOM / Educational innovation ¿Does the protagonist fail?: the ESCOM case. RIDE Revista Iberoamericana para la Investigación y el Desarrollo Educativo, v.8, n.15, pp. 710-734. https://doi.org/10.23913/ ride.v8i15.317 
La Plata, C. (2014). Errores en torno a la comprensión de la definición de límite finito de una función real de variable real. Tesis de maestría no publicada, Pontificia Universidad Católica del Perú.

Lillo. (2012). Actualización en el diagnóstico y manejo de la Infección Urinaria en pediatría. Revista chilena de pediatría, v.8, n. 3, pp. 269-278.

Mattos. (2018). Un análisis de las concepciones acerca de las dificultades, los obstáculos y los errores relativos al límite. (Trabajo de grado de maestría). Pontificia Universidad Católica del Perú Escuela de Posgrado.

MEN. (2003). Estándares Básicos de Competencias Ciudadanas. MEN: Bogotá.

Olivares-Parada, G., Olivares-Parada, P., y Parada-Rico, D. (2021). El contexto de la COVID-19 como espacio para repensar la virtualización educativa por parte de docentes universitarios. Educación y Humanismo, v.23, n.40, 1-17. https://doi. org/10.17081/eduhum.23.40.4276

Padilla-Escorcia, I. y Conde-Carmona, R. (2020). Uso y formación en TIC en profesores de matemáticas: un análisis cualitativo. Revista Virtual Universidad Católica del Norte, n. 60, 116-136. https:// www.doi.org/10.35575/rvucn.n60a7

Palomo, R., Ruiz, J., y Sanchez, J. (2006). Las TIC como agentes de innovación educativa. Junta de Andalucía, Consejería de Educación Dirección General de Innovación Educativa y Formación del Pro- fesorado. España. https://redined.educacion.gob. es/xmlui/handle/11162/3046

Paniagua, E. (2015). Metodología para la validación de una escala o instrumento de medida. Facultad Nacional de Salud Pública, 1-5.

Porlán, R. (2020) El cambio de la enseñanza y el aprendizaje en tiempos depandemia. Revista de Educación Ambiental y Sostenibilidad, v. 2, n.1, 1502. https://doi.org/10.25267/Rev_educ_ambient_sostenibilidad.2020.v2.i1.1502

Radillo, M., y González, L. (2014). Enseñanza del concepto de límite de una función mediante sus diversas representaciones semióticas, a nivel licenciatura. En P. Lestón (Ed.), Acta Latinoamericana de Matemática Educativa. Comité Latinoamericano de Matemática Educativa. pp. 853-861.

Rodríguez Areal, E., Pérez, M. A., Fernández, A., Martín, L., y Guevara, R. (2014). Una experiencia en el empleo de las TIC en la enseñanza de la Matemática. Congreso Iberoamericano de Ciencia, Tecnología, Innovación y Educación.

Stake, R. (2010). Qualitative research. Studying how things work. The Gilford Press, 88-91.

Trujillo, J. A., Vera, C. L. y Sosa, D. F. (2019). Ingeniería didáctica como recurso metodológico para el aprendizaje de los conceptos de límite y continuidad. Perspectivas, v.4, n.1, 39-47. https://doi. org/10.22463/25909215.1758 
UNESCO (2020). Iniciativas de aprendizaje a distancia basadas en uso de internet y otros medios de comunicación. SITEAL. https://www.siteal.iiep. unesco.org/respuestas_educativas_covid_19

Valbuena, S., Conde-Carmona, R. y Ortiz, J. (2018). La Investigación en educación matemática y Práctica Pedagógica, perspectiva de licenciados en Matemáticas en formación. Revista Educación y Humanismo, v.20, n. 34, pp. 201-215. http://doi. org/10.17081/eduhum.20.34.2593

Valbuena, S., Conde-Carmona, R., y Padilla-Escorcia, I. (2018). La práctica pedagógica en la investigación en educación matemática desde la perspectiva de los egresados. Universitas humanística, v. 86 , pp. $249-273$. https://doi.org/10.11144/Javeriana.uh86.ppie

Valbuena, S., y Conde-Carmona, R. (2018). Formación de profesores en Colombia. Saber, Ciencia y Libertad, v. 13, n. 2, pp. 226-236. https://doi. org/10.18041/2382-3240/saber.2018v13n2.4636
Van Hiele, P. (1957). El problema de la comprensión (en conexión con la comprensión de los escolares en el aprendizaje de la geometría. (Tesis doctoral no publicada). Universidad de Utrecht.

Vásquez, C., Ruz, F., y Martínez, M. (2020). Recursos virtuales para la enseñanza de la estadística y la probabilidad: un aporte para la priorización curricular chilena frente a la pandemia de la COVID-19. Tangram: Revista de Educação Matemática, v. 3, n. 2, pp. 159-183. https://doi.org/10.30612/ tangram.v3i2.12299

Volverás-Espinosa, A. (2015). Propuesta didáctica para la enseñanza de límites de funciones en el grado undécimo de la I.E el rosario integrando GeoGebra. (Trabajo de grado de maestría) Universidad Nacional de Colombia Sede Manizales Facultad De Ciencias Exactas y Naturales Departamento de Matemáticas y Estadística Manizales. https://repositorio.unal.edu.co/handle/unal/55982

Ynoub. (2015). La observación participante y no participante. Cuestión de método. CENGAGE Learning, 1-416. 